\title{
SMARThivVLmos: A complexity-free and cost effective dynamic model technology for monitoring HIV viral load in resource-poor settings
}

\author{
Aboubakar Yari ${ }^{1,{ }^{*}}$, Frederic S. Passo ${ }^{1}$, Venus Yari $^{1}$, Ousmane Sanni ${ }^{1}$, Myra Yari $^{1}$, Mathieu Z. Dovenou ${ }^{1}$, Rachide \\ Traore $^{1}$ and Jean-Pierre Hounyet ${ }^{2}$ \\ ${ }^{1}$ Biotech tropicana, Inc, Parakou, Benin; ${ }^{2}$ Hôpital Saint-Jean de Dieu, Boko, Benin ; Aboubakar Yari* - E-mail: ayari@biotechtropicana.com; \\ * Corresponding author
}

received December 18, 2007; revised January 14, 2008; accepted January 19, 2008; published January 27, 2008

\begin{abstract}
:
We design a "simple" and "low cost" model technology for monitoring HIV viral load in resource-poor settings: SMARThivVLmos. Cost and complexity are the major challenges to the developing world, in monitoring HIV patients viral load. We have previously demonstrated in our SMARThivPack model that cost and complexity of laboratory monitoring of HIV patients, may be reduced not only at a first technology development level, but also at a second technology implementation, and at a third global coordination levels. In our SMARThivPack model, the P24 HIV viral load monitoring system passed both the "cost" and the "complexity" tests. However, compared to other alternative viral monitoring systems such as the Cavidi EXAVIR, the sensitivity of the P24 system is too low. Here we describe a dynamic model technology that overcomes the sensitivity barrier of the P24 system while maintaining simplicity and low cost.
\end{abstract}

Keywords: HIV viral load measurement; HIV surveillance technologies; cost and complexity; resource-poor countries; alternative technologies

\section{Background:}

In the clinical management of HIV patients; CD4 count is used to determine when to start AntiRetroViral (ARV) treatment, and viral load data are used to monitor whether ARV treatment is successful. Viral load testing is routinely performed in the developed world. However, viral load monitoring was not recommended in the World Health Organisation (WHO) 2003 HIV patients treatment guidelines for resource-poor settings. [1, 2] In the developing world, equipments to perform viral load measurement are often unavailable, and where available the high cost of kits limited viral load testing in resource-poor settings.[2] Expert panels therefore recommended cheaper and simpler so called alternative technologies for viral load testing in resource-poor settings.[3, 4]

We have previously proposed a "three tests" combo kit model for monitoring HIV patients in resource-poor settings: SMARThivPack. [3] Our SMARThivPack model retained the alternative P24 HIV viral load monitoring system. The p24 system is both cheap and simple. The drawback of the p24 system is its low sensitivity. While the alternative Cavidi EXAVIR viral load testing system can detect down to 500 copies/ml of viral load, the cut-off value of the P24 system is 30.000 copies/ml. [4] However, incorporation of the Cavidi EXAVIR in our SMARThivPack model would raise equipment cost, because the system relied on different equipments than the ones used to perform CD4 count and pharmacoresistance testing. [3] Here we describe a dynamic model technology to improve the sensitivity, while maintaining simplicity and low cost, in monitoring HIV viral load in resource-poor settings.

\section{Description:}

We used web-based data mining and detailed investigation of existing viral load monitoring protocols, to design a model technology for monitoring HIV viral load in resource-poor settings. Using our cost and complexity level standards for guidance, we aim to design an HIV load measurement system that is compatible with our SMARThivPack model. [3] Our cost standard favours "low cost" over "high cost", and our complexity level standard favours "user friendly" "automated" text execution, over "manual" test execution. Our SMARThivPack model favours specialisation to a limited number of technologies, over technology and skill diversity.

In designing our dynamic SMARThivVLmos model, we hypothesize that the reliance of the P24 viral load measurement system ("P24 system") on ELISA plate reader makes the system compatible with our SMARThivPack model, and that the lower sensitivity of the P24 system may be overcome within our SMARThivPack model without further investment neither in equipment cost nor in training cost. Furthermore, compared to a single "static" technology system, the dynamic nature of our SMARThivVLmos expands the possibility of protocol choice for HIV viral load measurement, on case by case basis. 


\section{www.bioinformation.net}

\section{Views \& challenges}

In SMARThivVLmos, the P24 system passed the cost and complexity tests using ELISA plate reader as essential equipment. SMARThivVLmos overcomes the sensitivity barrier of the P24 system by incorporating a PCR-based viral load measurement system.

The P24 system passed the "cost” test in our SMARThivPack model with a total equipment cost of \$ 0 USD. Cost of the essential ELISA plate reader was applied toward the CD4 counting CAPCELLIA technology. [3] The p24 system also passed the "complexity level" test in our SMARThivPack model. ELISA is a simple technology that does not require long term training and viral load measurement is “automatically" performed by the ELISA plate reader. [3]

The drawback of the "simple" and "low cost" p24 system is its "low sensitivity". The cut off value of the p24 system is 30.000 copies $/ \mathrm{ml}$. Using the manufacturer provided reagents the p24 system can detect viral copies when the per/ml plasma viral content is 30.000 copies or above. [4, 5, 6], (Figure 1(a)) The Cavidi EXAVIR, an HIV viral load monitoring system recommended for resource-poor settings, has a cut off value of 500copies/ml. [7] Our SMARThivPack model did not retain the Cavidi Exavir technology because incorporation of the Cavidi EXAVIR system would raise both equipment cost and complexity, by adding the requirements of different equipments and skills. [3, 7] Therefore, based on our standard, the Cavidi EXAVIR systems failed both the cost test and the complexity level test. A technology must overcome both cost and complexity factors to be successful in resourcepoor and low expertise countries. However, the failure of a given technology to qualify within one "three tests" combo kit pack model does not necessarily mean that the same technology may not qualify within another pack model. Components of the Biotech tropicana, Inc SMARThivPack are dynamic structures. [3]

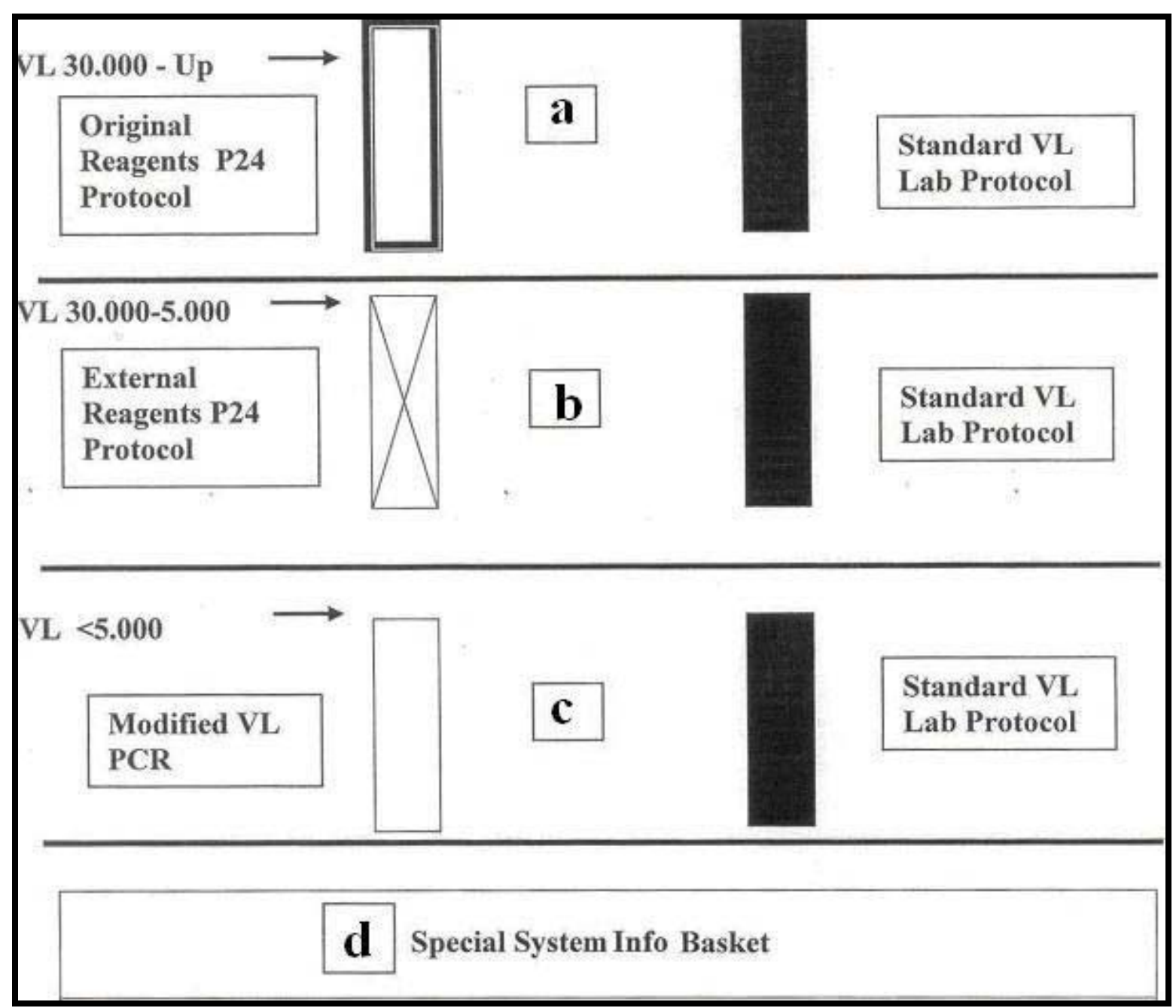

Figure 1: SMARThivVLmos: A conceptual framework. SMARThivVLmos is a dynamic HIV viral load monitoring model technology that comports three (c) protocols. Using the manufacturer provided reagents; the sensitivity of the P24 is satisfactory at 30.000 copies $/ \mathrm{ml}$ or above (a). Using external reagent the sensitivity may be improved down to 5.000 copies/ml. (b) Our model incorporated a "modified PCR-based" protocol for viral load values below 5.000 copies/ml. (c) Our SMARThivGLOBALmos system (under construction) incorporates at least one (a) control standard laboratory in reference centres, (Standard VL Lab Protocol) in addition to the alternative SMARThivPack laboratories in peripheral centres. We had a special info basket for recording special issues encountered while using the system and for recording improvements in the investigational areas. (d) 


\section{Bioinformation}

The sensitivity of the P24 system may be improved using reagents other than the ones provided by the manufacturer. It has been reported that using an external buffer for sample preparation, the sensitivity of the p24 system may be improved to 5000 copies/ml. [4, 8]; (Figure 1(b)) However, compared to the 500 copies/ml using the Cavidi system, the 5000 copies/ml using the P24 is still low.

To maintain "simplicity" and "low cost" while improving "sensitivity" we add one (1) additional protocol to our SMARThivVL system. Hamatake et al. reported a competitive PCR-based "simple" and "low cost" viral load system. [9] PCR-based viral load systems are generally ultra sensitive. [10] The PCR-based viral load system developed by Hamatake et al; can detect down to 110 copies/ml. [9] Our SMARThivPack model comports a PCR system for monitoring HIV drug resistance in resource-poor settings. [3] Because we applied the cost of the PCR equipments toward the drug resistance monitoring system, we can add a PCRbased viral load protocol without violating our "cost" standard. Because our SMARThivPack model comports a "PCR team" for performing HIV drug resistance, the addition of the modified-PCR protocol in our SMARThivVLmos system does not require a new skill, consistent with our skill diversity avoidance criteria. [3] (Figure 1(c))

The drawbacks of the PCR-based systems are their "high cost" and "high complexity" $[3,4,10]$ Most of these pcrbased systems failed both our cost and complexity tests, for the developing world. Our "cost" standard favour "low cost" over "high cost", and our "complexity level" standard favours "simple, user friendly" "automated" technologies over technologies that are "complex" and/or requires substantial human input. [3]

The Biotech tropicana, Inc SMARThivVLmos is a dynamic structure of the Biotech tropicana, Inc SMARThivPack model for improving HIV patients monitoring standards in resourcepoor settings. Our SMARThivVLmos system combines "low cost" and "simplicity" of the ELISA technology with "high sensitivity" of the PCR-based viral load systems. The flexibility of our SMARThivVLmos system permits choice between three protocols, on case by case basis. The Biotech tropicana, Inc SMARThivVLmos is an intended component of the Biotech tropicana, Inc SMARThivPack. A detailed protocol for test execution using the SMARThivVLmos technology is under development at Biotech tropicana, Inc.
The Biotech tropicana, Inc SMARThivPack is an intended component of the Biotech tropicana, Inc "life Box", comporting in addition to the "three tests" combo kit, an ELISA plate reader and a PCR set up. The Biotech tropicana, Inc "Life Box" is an intended component of the Biotech tropicana, Inc SMARThivGLOBALmos, a third level global coordination cost and complexity reductions model, comporting in addition to the SMARThivPack-based "Life Box", alternative "Life Boxes" (“A-Life Box"). Choosing from the pool of available alternatives technologies recommended for the developing world, Biotech tropicana, Inc is in the process of constructing alternative hivPack models ("A-Life Box"), for incorporation into the Biotech tropicana, Inc the SMARThivGLOBALmos. The Biotech tropicana, Inc “A-life Boxes” are dynamic structures, constructed on case by case basis, based on existing equipments and skills in a given laboratory, to overcome cost and complexity.

We design a dynamic model technology by combining the "simplicity" and "low cost" of the ELISA technology and the "high sensitivity" of a PCR-based HIV viral load monitoring system: SMARThivVLmos. Our dynamic SMARThivVLmos system is flexible enough to, permit protocol choice on case by case basis, for optimal cost/sensitivity ratio.

\section{References:}

[01] http://www.who.int/hiv/pub/prev_care/en/arvrevision2 003en.pdf

[02] http://www.journals.uchicago.edu/doi/pdf/10.1086/51 0073

[03] A. Yari, et al., Bioinformation, 2: 97 (2007)

[04] S. Fiscus, et al., PLoS Med., 10: 417 (2006) [PMID: 17032062]

[05] A. Pascual, et al., J Clin Microbiol., 40: 2472 (2002) [PMID 12089264]

[06] J. Lombart, et al., AIDS, 19:1273 (2005) [PMID 16052082]

[07] V. Greengrass, et al., Curr HIV Res., 3:183 (2005) [PMID 15853722]

[08] J. Schupbach, J. Acquir Immune Defic Syndr., 33: 292 (2003) [PMID 12843739]

[09] M. Hamatake, et al., J. Virol Methods, 142: 113 (2007) [PMID: 17367872]

[10] http://mednet2.who.int/sourcesprices/sp_1b.pdf

Edited by P. Kangueane Citation: Yari et al., Bioinformation 2(6): 246-248 (2008) License statement: This is an open-access article, which permits unrestricted use, distribution, and reproduction in any medium, for non-commercial purposes, provided the original author and source are credited. 